\title{
EXPERIMENTAL DEMONSTRATION OF PRODUCING HIGH RESOLUTION ZONE PLATES BY SPATIAL-FREQUENCY MULTIPLICATION*
}

\author{
W. B. Yun \\ Materials Science Division, Argonne National Laboratory \\ Argonne, It 60439 \\ and \\ M. R. Howells \\ Center for X-Ray OptIcs, Lawrence Berkeley Laboratory \\ Berkeley, CA 94720 \\ March 1988 \\ CONF-8708180--12

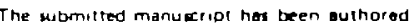 \\ U. S. Govarment purposes \\ DE88 012032
}

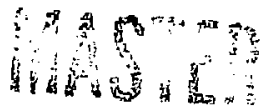

This paper submitted to the proceedings on "Soft X-Ray Microscopy Conference" at Brooknaven National Laboratory, August, 1987.

\section{DISCLAIMER}

\footnotetext{
This report was prepared as an account of work pansored by an agency ul ine lintled States Covernment. Neither the 1!nuted States Government nur any agency thereot. nur uny of thesr emplayees, makes any uarranty, express ur mpled, ar assumes any legal hahilat ur cenponsi-

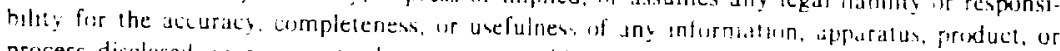
process disclosed, of represents that ils use would not infringe pribitely ansied rights. Refer-

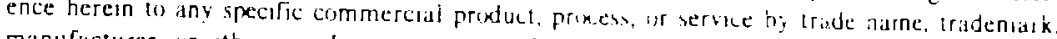
manufactures, or otherwise does not necessarily combtitute or ampl: its endursement. recom. mendatior. or favoring by the Lnited States Guvernment or any angency thereof The views and opinions of authors expressed herein do not necessarily slate or reflect those of the United States Government or any agency thereof
}

\footnotetext{
FThis work supported by the U.S. Department of Energy, BES-Materials Sciences, under Contract W-31-109-ENG-38.
} 
Experimental Demonstration of Producing High Resolution Zone Plates by SpatialFrequency Multiplication

W B. Yurt and M. R. Howells

Argonne National Laboratory. Argonre. IL 60439: Center for X-Ray Optics. Lawrence Berkeley Laboratory. Berkeley. California 94720.

\section{INTRODUCTION}

In an earlier publication [1]. the possibility of producing high resolution zone plates for $X$ ray applications by spatial-frequency multiplication was analyzed theoretically. The theory predicted that for a daughter zone plate generated from the Interference of mth and $n t h$ diffraction orders of a parent zone plate. Its primary focal spot size and focal length are one $(m+n) t h$ of their rounterparts of the parent zone plate. respectively. It was also shown that a zone plate with the outermost zone width of as small as $13.8 \mathrm{~nm}$ might be produced by this technique. In this paper. we report an experiment which we carrled out with laser light $(\lambda=1100 \AA)$ for demonstrating this technique. In addition, an outlook for producing high resolution zone plates for $X$-ray application is brlefly discussed.

\section{THE EXPERIMENTAL DEMONSTRATION}

The demonstration can he logically divided into two parts. In the first part, an interference pattern of two diffraction orders of a parent zone plate was recorded. In the second part. the primary focus of the recording (daughter zone plate) was recorded and its focal length was measured. Satisfactory demonstration was thought to have been made when the measured focal spot sizes and focal lengths of several daughter zone plates (each generated from different combination of $m$ and $n$ ) were in good agreement with those predicted by theory.

Table 1 Parameters of the parent zone plate

focal length

radius of inner stop

radius of the outer stop

number of open zones

maximum zone index

zonewldth of the outer most zone $f(4165 \mathrm{~A})=59 \mathrm{~cm}$

$3.56 \mathrm{~mm}$

$4.86 \mathrm{~mm}$

24

96

$25.3 \mu \mathrm{m}$

The arrangement of optics used in recording the daughiter zone plates is shown In Fig. 1. The parent zone plate used was one demagnified from an original zone plate drawn by means of a computer plotter. Some relevant parameters of the parent zone plate are listed in Table 1. The Inner stop was purposely made to make the two-beam Interference possible [1]. A laser beam magnified by the beam expander to a size larger than the size of the parent zone plate was used for lliumination. The interference pittern of the mth and nth 
order diffractions. whose intensity distribution follows the zone plate geometry, was recorded on high resolution holographic film plate (llford type TE). which had a resolving power of 3000 lines/mm.

Annular region of meh and neh order interference

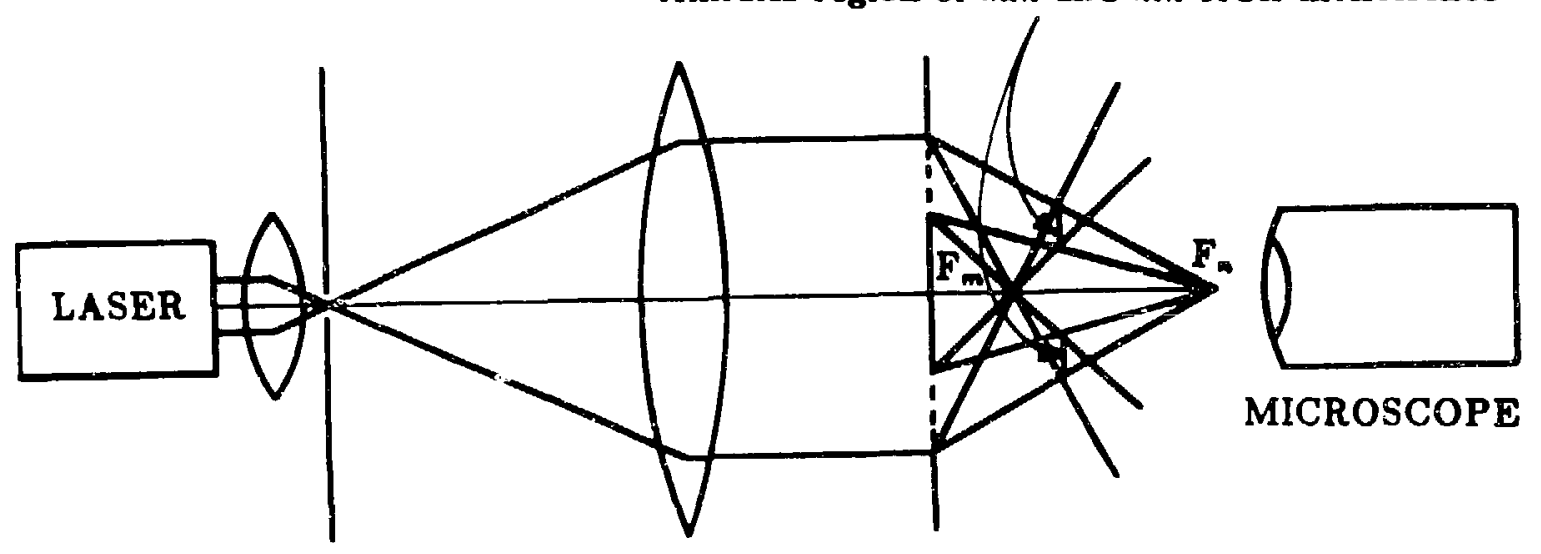

BEAM EXPANDER

PARENT ZONE PLATE

Fig. 1 Scti:umatic illustration of the arrangement used in recording the daughter zone plates. The microscope aids in the inspection and accurate location of the daughter zone plate patterns.

With the help of the microscope (Fig. 1). the intensity distribution of the optical field behind the parent zone plate (e.g.. the focl of the parent zone plate and the interference patterns) could be visually studied. Sharp focusing was observed for the focl $F_{1}, F_{2}, F_{3}$. $F_{4}$ and $F_{b}$. For foci with diffraction order higher than 6 . aberrations increased quickly with the diffraction order. For foci with diffraction order higher than 8 , aberrations became so strong that a clear focal spot could not be observed. This is probably related to the degree of imperfection of the parent zone plate. The observed results may be explained with the knowledge that the requirements on the degree of perfection of a zone plate (e.g. the accuracy of placement of zone positions and the circularity of zones of the zone plate) for producing good focus increases with diffraction order.

The scale on the optical bench. on which the observing microscope could slide back and forth. was used for measuring distances of interest. The focal length of the parent zone plate was measured to be $59 \pm .5 \mathrm{em}$. which was In good agreement with that calculated (Table 1). A daughter zone plate corresponding to the $m t h$ and $n t h$ order interference pattern was recorded by placing the emulsion side of the recording film plate at the theoretical distance. $2 f /(n+m)$ away from the parent zone plate [1]. Exposure time was properly set to obtain optimal fringe visibility in the daughter zone plate.

For measuring the focal length of a daughter zone plate and recording its primary focus on a film plate. the same arrangement as that shown in Fig. 1 was used except where the parent zone plate was replaced by the daughter zone plate. In Table 2. the measured focal lengths of several daughter zone plates together with those predicted by theory are listed. The notation n\&m in the column under daughter zone plate is used to denote that the corresponding daughter zone plate was generated from the Interference of $m$ th and $n$th 
diffracti-n orders of the parent zone plate. The error in the measurement of the focal lengths was mainly due to the rough scale on the optical bench. The recorded primary foci of the daughter zone plates are shown in Fig. 2b. For convenlence of comparison. the microphotographs shown in Fig. 2 were arranged so that the focl in the same column should have the same focal spot size according to the theory. All the microphotographs were magnified $320 \mathrm{t} / \mathrm{mes}$ from the original recordings for convenience of observation

Table 2 Focal lengths of the daughter zone plates

\begin{tabular}{lll} 
Daughter & \multicolumn{2}{c}{ Focal Length } \\
Zone PLate & Measured $(\mathrm{mm})$ & Theory $(\mathrm{mm})$ \\
$0 \& 2$ & $29.5 \pm .5$ & 29.5 \\
$0 \& 3$ & $19.5 \pm .5$ & 19.6 \\
$0 \& 4$ & $14.5 \pm .5$ & 14.7 \\
$0 \& 5$ & $12.0 \pm .5$ & 11.8 \\
$1 \& 2$ & $19.5 \pm .5$ & 19.6 \\
$1 \& 3$ & $14.5 \pm .5$ & 14.7 \\
$1 \& 4$ & $12.0 \pm .5$ & 11.8 \\
$-1 \& 4$ & $19.5 \pm .5$ & 19.6 \\
$2 \& 3$ & $12.0 \pm .5$ & 11.8 \\
$3 \& 7$ & $6.0 \pm .5$ & 5.9 \\
$4 \& 6$ & $6.0 \pm .5$ & 5.9
\end{tabular}

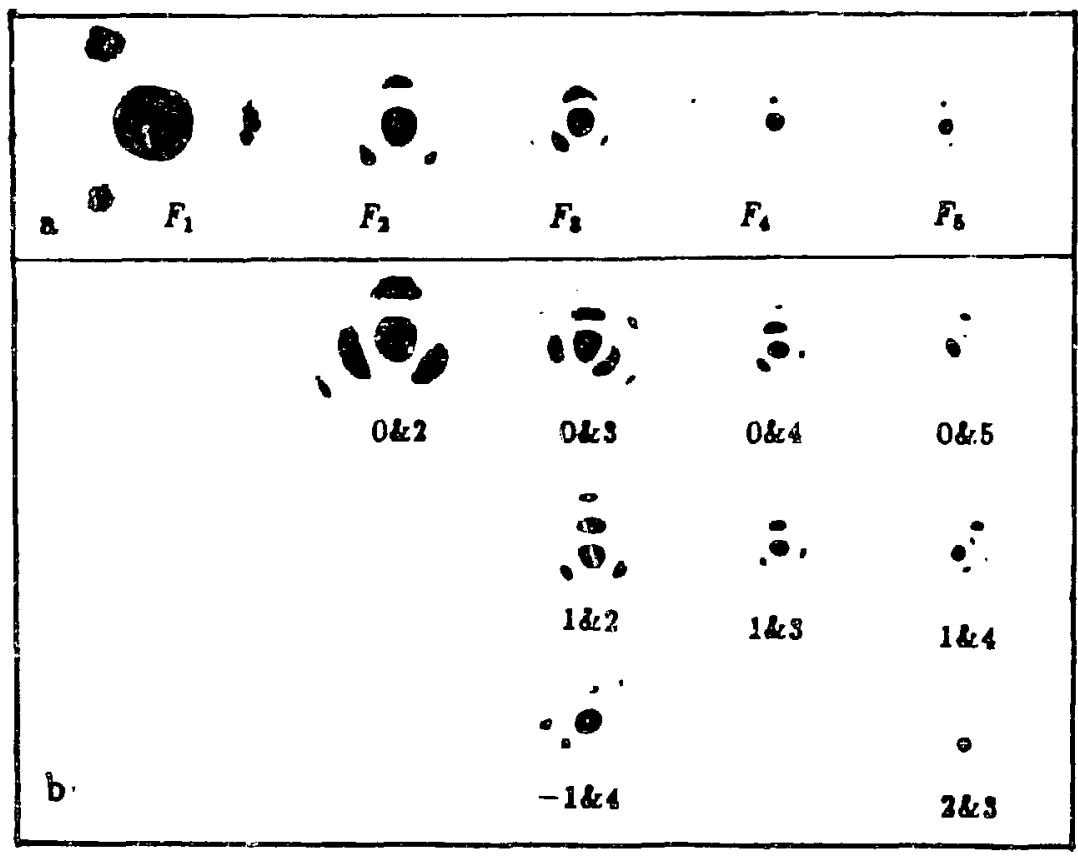

Fig. 2 Comparison of the foci of the parent zone piate (a) with the primary focus of its daughter zone plates (b). The $F_{i}$ in (a) is used to denote the ith focus of the parent zone plate. The notation n\&s in (b) indicates that the corresponding daughter zone plate was obtained through nth and mith order interference. 
We see from Fig. 2 that quality of primary focl of the daughtei cuice piates resulting from $0 \& 2.0 \& 3.0 \& 4.1 \& 2.1 \& 3$ and $1 \& 4$ are fairly good. Note that all foci In a column have similar size. as expected from the theoretical analysis. Due to limitation in our setup. the foci of the daughter zone plates resulting from 3rd \& 7th order interference and 4th \& 6th order interference were not recorded. Sharp focusing from those two daughter zone plates, however, was observed with the microscope. Recalling that the 10th order focus of the parent zone plate suffers very severe degradation. we think that a defect cancelation effect may have played some role in the generation of the daughter zone plates. In other words, even if two foci Involved in generating a daughter zone plate have some defects. the daughter zone plate generated may still have good focusing property. In addition, we also notice that the foci involved in generating the two zone plates have less aberration than the 10th order focus

It is interesting to note the similarity between the process of our demonstration and the process of hologram recording and reconstruction. However, except for the daughter zone plates generated from zeroth order and $m$ th order interference. the Illuminating beam in our demonstration. which can be viewed as the reconstruction wave in hologram reconstruction. is different from that used at recording. where one of the two interfering beams has served as the reference beam. This leads to the results that the primary focal spot of a daughter zone piate recorded from zeroth order and $m$ th order interference is the same as the $m$ th order focus of the parent zone plate. while the primary focus of the other daughter zone plates is smaller than any one of the foci involved. The demagnification in the latter case is a well known result in holography.

\section{SUMMARY}

The principle of the techique is experimentally demonstrated. Improvement in zone plate spatial resolution up to a factor of 10 was achieved. In order to apply the technique to produce zone plates with spatial resolution down to the level of $10 \mathrm{~nm}$. it is neccessary to use a $X$-ray source of adequate coherent flux for illumination. With some positive findings from the case study ${ }^{2}$ of a soft $X$-ray version of the experiment described in this context. we believe that the technique may ultimately play an important role in producing high resolution zone plates for X-ray application.

\section{REFERENCES}

1. W.B. Yun and M.R. Howells: J. Opt. Soc. Am. A. 4(1). 34(1987).

2. W.B. Yun: Ph. D. thesis (State University of New York at Stony Brook. 1987).

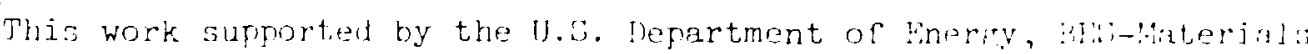
Sciences, undor contract W-31-100-Enli-38. 